\title{
Learning Graph Quantization
}

\author{
Brijnesh J. Jain, S. Deepak Srinivasan, \\ Alexander Tissen, and Klaus Obermayer \\ Berlin Institute of Technology, Germany \\ jbj@cs.tu-berlin.de
}

\begin{abstract}
This contribution extends learning vector quantization to the domain of graphs. For this, we first identify graphs with points in some orbifold, then derive a generalized differentiable intrinsic metric, and finally extend the update rule of LVQ for generalized differentiable distance metrics. First experiments indicate that the proposed approach can perform comparable to state-of-the-art methods in structural pattern recognition.
\end{abstract}

\section{Introduction}

Learning vector quantization (LVQ) as introduced by Kohonen [1] is a supervised learning algorithm for pattern classification. To classify patterns, LVQ applies the nearest neighbor rule using a condensed set of prototypes. Prototypes are learned by combining competitive learning with supervision. LVQ is easy to implement, runs efficiently, allows to control the complexity of the resulting classifier, naturally deals with multiclass problems, provides an interpretable rather than a block-box model, and in many cases provides state of the art performance.

LVQ and related methods have been originally devised for feature vectors equipped with the Euclidean metric. Extensions have been proposed, for example, for vectors with arbitrarily differentiable distance functions [8, for variable length and warped feature sequences [18, and for strings 12. But there have been no efforts reported towards extending LVQ for the domain of attributed graphs, although there are a number of related unsupervised methods that extend competitive learning for central clustering to structured data [7669],

In this contribution, we generalize LVQ to learning graph quantization (LGQ). The challenge consists in formulating an update rule for prototype adaption. For differentiable metrics on vectors such as the Euclidean metric, adaption amounts in moving prototypes along the line determined by the gradient of the underlying distance metric. Graph distance metrics, however, are not differentiable in general and therefore local gradient information is unavailable for prototype adaption. To overcome this problem, an appropriate approach to represent graphs is necessary. As such an approach, we suggest to represent graphs as points in some Riemannian orbifold. An orbifold is a quotient of a manifold by a finite group action. Using orbifolds, we derive an intrinsic metric that enables us to adopt concepts such as the derivative and gradient. Since the intrinsic metric 
of a graph orbifold is generalized differentiable, we can apply local gradient information for adapting graph prototypes almost everywhere. At this point, it is important to note that the intrinsic metric is not an artificial construction for analytical purposes but rather a common choice of graph distance metric in a number of applications [1/2 3|5|19|21]. Experiments on three data sets of the IAM graph database [15] show that the proposed LGQ approach returns state-of-the-art results.

The approach presented in this contribution can be applied to finite combinatorial structures other than graphs such as, for example, point patterns, sequences, trees, and hypergraphs. For the sake of concreteness, we restrict our attention exclusively to the domain of graphs. For graphs consisting of a single vertex with feature vectors as attributes, the proposed learning graph quantization (LGQ) reduces to LVQ.

This paper is organized as follows. Section 2 represents graphs as point in some orbifold. Section 3 extends LVQ to LGQ. In Section 4, we present and discuss experiments. Finally, Section 5 concludes.

\section{Graph Orbifolds}

Crucial for designing pattern classification algorithms is an appropriate representation of the data space. We suggest to represent attributed graphs as points in some graph orbifold. A graph orbifold is the simplest form of a Riemannian orbifold. For proofs of the statements in this section we refer to [10].

\subsection{Representation of Attributed Graphs}

Let $\mathbb{E}=\mathbb{R}^{d}$ be a Euclidean space. An attributed graph is a triple $X=(V, E, \alpha)$ consisting of a set $V$ of vertices, a set $E \subseteq V \times V$ of edges, and an attribute function $\alpha: V \times V \rightarrow \mathbb{E}$, such that $\alpha(i, j) \neq \mathbf{0}$ for each edge and $\alpha(i, j)=\mathbf{0}$ for each non-edge. Attributes $\alpha(i, i)$ of vertices $i$ may take any value from $\mathbb{E}$.

For simplifying the mathematical treatment, we assume that all graphs are of order $n$, where $n$ is chosen sufficiently large. Graphs of order less than $n$, say $m<n$, can be extended to order $n$ by including isolated vertices with attribute zero. For practical issues, it is important to note that limiting the maximum order to some arbitrarily large number $n$ and extending smaller graphs to graphs of order $n$ are purely technical assumptions to simplify mathematics. For pattern recognition problems, these limitations should have no practical impact, because neither the bound $n$ needs to be specified explicitly nor an extension of all graphs to an identical order needs to be performed. When applying the theory, all we actually require is that the graphs are finite.

A graph $X$ is completely specified by its matrix representation $\boldsymbol{X}=\left(\boldsymbol{x}_{i j}\right)$ with elements $\boldsymbol{x}_{i j}=\alpha(i, j)$ for all $1 \leq i, j \leq n$. By concatenating the columns of $\boldsymbol{X}$, we obtain a vector representation $\boldsymbol{x}$ of $X$.

Let $\mathcal{X}=\mathbb{E}^{n \times n}$ be the Euclidean space of all $(n \times n)$-matrices with elements from $\mathbb{E}$ and let $\mathcal{T}$ denote a subgroup of all $(n \times n)$-permutation matrices. Two 
matrices $\boldsymbol{X}, \boldsymbol{X}^{\prime} \in \mathcal{X}$ are said to be equivalent, if there is a permutation matrix $P \in \mathcal{T}$ such that $\boldsymbol{P}^{\top} \boldsymbol{X} \boldsymbol{P}=\boldsymbol{X}^{\prime}$. The quotient set

$$
\mathcal{X}_{\mathcal{T}}=\mathcal{X} / \mathcal{T}=\{[\boldsymbol{X}]: \boldsymbol{X} \in \mathcal{X}\}
$$

consisting of all equivalence classes $[\boldsymbol{X}]$ is a graph orbifold over the representation space $\mathcal{X}$. Its orbifold chart is the surjective continuous mapping

$$
\pi: \mathcal{X} \rightarrow \mathcal{X}_{\mathcal{T}}, \quad \boldsymbol{X} \mapsto[\boldsymbol{X}]
$$

that projects each point $\boldsymbol{X}$ to its orbit $[\boldsymbol{X}]$. A graph orbifold is the simplest form of a so called Riemannian orbifold.

In the following, we identify $\mathcal{X}$ with $\mathbb{E}^{N}\left(N=n^{2}\right)$ and consider vector- rather than matrix representations of abstract graphs. We use capital letters $X, Y, Z, \ldots$ to denote graphs from $\mathcal{X}_{\mathcal{T}}$ and write $\boldsymbol{x} \in X$ if $\boldsymbol{x}$ is a vector representation that projects to $X$ (i.e. if $\pi(\boldsymbol{x})=X$ ). Since $\mathbb{E}$ is Euclidean so is $\mathcal{X}$. By $\|\cdot\|$ we denote the Euclidean norm defined on $\mathcal{X}$.

\subsection{Generalized Differentiable Graph Metric}

Next, we introduce and analyze an intrinsic metric structure on graph orbifolds. For graphs with discrete attributes the intrinsic metric is related to the concept of maximum common subgraph.

The Graph Metric. We consider graph metrics of the form

$$
d(X, Y)=\min \left\{\|\boldsymbol{x}-\boldsymbol{y}\|^{2}: \boldsymbol{x} \in X, \boldsymbol{y} \in Y\right\} .
$$

A pair $\boldsymbol{x}, \boldsymbol{y} \in X \times Y$ is an optimal alignment if $d(X, Y)=\|\boldsymbol{x}-\boldsymbol{y}\|^{2}$. By $\mathcal{A}(X, Y)$ we denote the set of all optimal alignments of $X$ and $Y$.

Since $\mathcal{T}$ is a group, we have

$$
d_{X}(Y)=\min \left\{\|\boldsymbol{x}-\boldsymbol{y}\|^{2}: \boldsymbol{y} \in Y\right\}=d(X, Y),
$$

where $\boldsymbol{x} \in X$ is an arbitrary vector representation. Hence, the graph distance $d(X, Y)$ can be determined by fixing an arbitrary vector representation $\boldsymbol{x} \in X$ and then finding a vector representation $\boldsymbol{y}_{*} \in Y$ that minimizes $\|\boldsymbol{x}-\boldsymbol{y}\|^{2}$ over all vector representations $\boldsymbol{y} \in Y$. Note that we also have $d_{X}(Y)=d(Y, X)$ by symmetry.

Generalized Differentiability. The lift $\tilde{d}_{X}$ of the parametrized graph distance function $d_{X}$ is defined by

$$
\tilde{d}_{X}: \mathcal{X} \rightarrow \mathbb{R}, \quad \boldsymbol{y} \mapsto \min \left\{\left\|\boldsymbol{x}-\boldsymbol{y}^{\prime}\right\|^{2}: \boldsymbol{y}^{\prime} \in Y\right\}
$$

where $\boldsymbol{x} \in X$ is an arbitrary vector representation. Certainly, the lift $\tilde{d}_{X}$ satisfies $\tilde{d}_{X}=d_{X} \circ \pi$ and is invariant under group actions of $\mathcal{T}$, that is $\tilde{d}_{X}(\boldsymbol{y})=\tilde{d}_{X}(\gamma(\boldsymbol{y}))$ for all $\gamma \in \mathcal{T}$. 
By lifting the distance function $d_{X}$ to the Euclidean space $\mathcal{X}$, we are in the position to transfer analytical concepts such as differentiability and gradients to functions on graph orbifolds. We say, the function $d_{X}$ is continuous (locally Lipschitz, differentiable, generalized differentiable) at point $Y \in \mathcal{X}_{\mathcal{T}}$ if its lift $\tilde{d}_{X}$ is continuous (locally Lipschitz, differentiable, generalized differentiable) at some vector representation $\boldsymbol{y} \in Y$ 1 This definition is independent of the choice of vector representations that project to $X$ and $Y$.

As a minimizer of a set of continuously differentiable distance functions, the function $d_{X}$ is generalized differentiable at any point $Y$. Though $d_{X}$ is not differentiable, it is locally Lipschitz and therefore differentiable almost everywhere.

Gradients. Suppose that $d_{X}$ is differentiable at $Y$ and let $\boldsymbol{x} \in X$ be arbitrary. Then the lift $\tilde{d}_{X}$ is differentiable at any vector representation that projects to $Y$. The gradient $\nabla \tilde{d}_{X}(\boldsymbol{y})$ of $\tilde{d}_{X}$ at $\boldsymbol{y}$ is of the form

$$
\nabla \tilde{d}_{X}(\boldsymbol{y})=-2\left(\boldsymbol{x}-\boldsymbol{y}_{*}\right)
$$

where $\left(\boldsymbol{x}, \boldsymbol{y}_{*}\right) \in \mathcal{A}(X, Y)$ is an optimal alignment. Since $d_{X}$ is differentiable at $Y$, the optimal alignment $\left(\boldsymbol{x}, \boldsymbol{y}_{*}\right)$ is unique. From

$$
\nabla \tilde{d}_{X}(\gamma(\boldsymbol{y}))=\gamma\left(\nabla \tilde{d}_{X}(\boldsymbol{y})\right)
$$

for all $\gamma \in \mathcal{T}$ follows that the gradients of $\tilde{d}_{X}$ at $\boldsymbol{y}$ and $\gamma(\boldsymbol{y})$ are vector representations of the same graph. Hence, at differentiable points $Y$, the gradient of $d_{X}(Y)$ at $Y$ is defined by the projection

$$
\nabla d_{X}(Y)=\pi\left(\nabla \tilde{d}_{X}(\boldsymbol{y})\right)
$$

of the gradient $\nabla \tilde{d}_{X}(\boldsymbol{y})$ at vector representation $\boldsymbol{y} \in Y$. Thus, the gradient of $d_{X}$ at $Y$ is a well-defined graph pointing to the direction of steepest ascent.

Generalized Gradients. Now suppose that $d_{X}$ is generalized differentiable at $Y$. Then the lift $\tilde{d}_{X}$ is generalized differentiable at any vector representation that projects to $Y$. The subdifferential $\partial \tilde{d}_{X}(\boldsymbol{y})$ of $\tilde{d}_{X}$ at $\boldsymbol{y}$ is a convex set containing

$$
-2\left(\boldsymbol{x}-\boldsymbol{y}_{*}\right) \in \partial \tilde{d}_{X}(\boldsymbol{y})
$$

as generalized gradient, where $\left(\boldsymbol{x}, \boldsymbol{y}_{*}\right) \in \mathcal{A}(X, Y)$ is an optimal alignment. From

$$
\partial \tilde{d}_{X}(\gamma(\boldsymbol{y}))=\gamma\left(\partial \tilde{d}_{X}(\boldsymbol{y})\right)
$$

for all $\gamma \in \mathcal{T}$ follows that the subderivatives of $\tilde{d}_{X}$ at $\boldsymbol{y}$ and $\gamma(\boldsymbol{y})$ project to the same subset of graphs. Hence, at generalized differentiable points $Y$, the subderivative of $d_{X}(Y)$ at $Y$ is defined by the projection

$$
\partial d_{X}(Y)=\pi\left(\partial \tilde{d}_{X}(\boldsymbol{y})\right)
$$

\footnotetext{
${ }^{1}$ Appendix $\mathrm{A}$ defines generalized differentiable functions.
} 
of the subderivative $\nabla \tilde{d}_{X}(\boldsymbol{y})$ at an arbitrary vector representation $\boldsymbol{y} \in Y$. Thus, the subderivative of $d_{X}$ at $Y$ is well-defined and coincides with the gradient at differentiable points, that is $\partial d_{X}(Y)=\left\{\nabla d_{X}(Y)\right\}$.

\section{Learning Graph Quantization}

The task of Learning Graph Quantization (LGQ) is to construct a classifier $c: \mathcal{X}_{\mathcal{T}} \rightarrow \mathcal{C}$ that maps graphs from $\mathcal{X}_{\mathcal{T}}$ to class labels from a finite set $\mathcal{C}$. The classifiers are parameterized by a set of $k$ prototypes $Y_{1}, \ldots, Y_{k} \in \mathcal{X}_{\mathcal{T}}$ with class labels $c_{1}, \ldots, c_{k} \in \mathcal{C}$. We predict the class label $c(X)$ of a new graph $X \in \mathcal{X}_{\mathcal{T}}$ by assigning it to the class label of the closest prototype according to the nearest neighbor rule. The goal of learning is to find a set of $k$ prototypes that best predicts the class labels of graphs from $\mathcal{X}_{\mathcal{T}}$. In the following, we extend LVQ and LVQ2.1 to the domain of graph orbifolds.

\section{$3.1 \quad$ LGQ}

Suppose that $\mathcal{S}=\left\{\left(X_{i}, y_{i}\right)\right\}_{i=1}^{n} \subseteq \mathcal{X}_{\mathcal{T}} \times \mathcal{C}$ is a training set consisting of $n$ input graphs $X_{i} \in \mathcal{X}_{\mathcal{T}}$ together with class labels $y_{i} \in \mathcal{C}$. The algorithm first chooses $k$ prototypes $\mathcal{Y}=\left\{\left(Y_{j}, c_{j}\right)\right\}_{j=1}^{k}$ such that each class is represented by at least one prototype. Next, during adaption, the algorithm randomly choses an example $(X, y) \in \mathcal{S}$ from the training set and modifies the closest prototype $Y_{X}$ in accordance with the current example. The input graph $X$ attracts its closest prototype $Y_{X}$ if the class labels $y$ of $X$ and $c_{X}$ of $Y_{X}$ agree. Otherwise, if the class labels differ, the input $X$ repels the closest prototype $Y_{X}$. To determine the closest prototype, LGQ applies the nearest neighbor rule

$$
Y_{X}=\arg \min _{Y \in \mathcal{Y}}\{d(X, Y)\} .
$$

To update the closest prototype $Y_{X}$, the algorithm fist selects an optimal alignment $\left(\boldsymbol{x}, \boldsymbol{y}_{\boldsymbol{x}}\right) \in \mathcal{A}(X, Y)$. Then it applies the standard LVQ update rule

$$
\boldsymbol{y}_{\boldsymbol{x}} \leftarrow\left\{\begin{array}{ll}
\boldsymbol{y}_{\boldsymbol{x}}+\eta\left(\boldsymbol{x}-\boldsymbol{y}_{\boldsymbol{x}}\right) & : y=c_{x} \\
\boldsymbol{y}_{\boldsymbol{x}}-\eta\left(\boldsymbol{x}-\boldsymbol{y}_{\boldsymbol{x}}\right) & : \quad y \neq c_{x}
\end{array},\right.
$$

where $\eta$ is a monotonically decreasing learning rate following the guidelines of stochastic optimization. The updated vector representation projects to the updated graph prototype. This process continues until the procedure satisfies a termination criterion. Algorithm 1 summarizes the LGQ procedure.

\subsection{LGQ2.1}

In contrast to LGQ the LGQ2.1 procedure updates the two closest prototypes $Y_{X}^{1}$ and $Y_{X}^{2}$ in accordance to the current training example $(X, y) \in \mathcal{S}$. The algorithm adapts the prototypes $Y_{X}^{1}$ and $Y_{X}^{2}$ if the following conditions hold: 
Algorithm 1. Learning Graph Quantization

\section{Input:}

training set $\mathcal{S}=\left\{\left(X_{1}, y_{1}\right), \ldots,\left(X_{n}, y_{n}\right)\right\} \subseteq \mathcal{X}_{\mathcal{T}} \times \mathcal{C}$

Procedure:

1. choose initial prototypes $\mathcal{Y}=\left\{\left(Y_{1}, c_{1}\right), \ldots,\left(Y_{k}, c_{k}\right)\right\} \subseteq \mathcal{X}_{\mathcal{T}} \times \mathcal{C}$

2. choose vector representations $\boldsymbol{y}_{1} \in Y_{1}, \ldots, \boldsymbol{y}_{k} \in Y_{k}$

3. repeat until termination

3.1. randomly select a training example $(X, y) \in \mathcal{S}$

3.2. let $Y_{X}=\arg \min _{Y \in \mathcal{Y}} d(X, Y)$

3.3 choose optimal alignment $\left(\boldsymbol{x}, \boldsymbol{y}_{\boldsymbol{x}}\right) \in \mathcal{A}\left(X, Y_{X}\right)$

3.4. determine learning rate $\eta>0$

3.5. update according to the rule

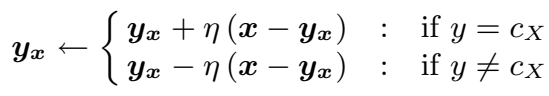

Return: set $\mathcal{Y}$ of prototypes

1. Exactly one of both prototypes $Y_{X}^{1}$ and $Y_{X}^{2}$ has the same class label as $X$

2. The input graph $X$ falls in a window around the decision border defined by

$$
\frac{d\left(X, Y_{X}^{2}\right)}{d\left(X, Y_{X}^{1}\right)}>\frac{1-w}{1+w}
$$

where $w$ is the relative width of the window.

For each prototype LGQ2.1 uses the same update rule as LGQ.

\section{Experiments}

To assess the performance of the proposed LGQ algorithms, we conducted first experiments.

\subsection{Data}

We selected four data sets described in [15. Each data set is divided into a training, validation, and a test set. The description of the data sets are mainly excerpts from 15. Table 1 provides a summary of the main characteristics of the data sets.

Letter Graphs (high distortion level). The letter data set compiles distorted letter drawings from the Roman alphabet that consist of straight lines only (A, E, F, H, I, K, L, M, N, T, V, W, X, Y, Z). The graphs are uniformly distributed over the 15 classes (letters). The letter drawings are obtained by distorting prototype letters at high distortion level. Lines of a letter are represented by edges and ending points of lines by vertices. Each vertex is labeled with a two-dimensional 
Table 1. Summary of main characteristics of the data sets

\begin{tabular}{lcccccc}
\hline \hline data set & \#(graphs) & \#(classes) & avg(nodes) & max(nodes) & avg(edges) & max(edges) \\
\hline letter & 750 & 15 & 4.7 & 8 & 3.1 & 6 \\
grec & 528 & 22 & 11.5 & 24 & 11.9 & 29 \\
fingerprint & 900 & 3 & 8.3 & 26 & 14.1 & 48 \\
\hline \hline
\end{tabular}

vector giving the position of its end point relative to a reference coordinate system. Edges are labeled with weight 1.

GREC Graphs. This data set 4] consists of graphs representing symbols from architectural and electronic drawings. The images occur at five different distortion levels. Depending on the distortion level, either erosion, dilation, or other morphological operations are applied. The result is thinned to obtain lines of one pixel width. Finally, graphs are extracted from the resulting denoised images by tracing the lines from end to end and detecting intersections as well as corners. Ending points, corners, intersections and circles are represented by vertices and labeled with a two-dimensional attribute giving their position. The vertices are connected by undirected edges which are labeled as line or arc. An additional attribute specifies the angle with respect to the horizontal direction or the diameter in case of arcs.

Fingerprint Graphs. This data set represents fingerprint images of the NIST-4 database [20] from four classes arch, left, right, and whorl. Fingerprint images are converted into graphs by filtering the images and extracting regions that are relevant [13. Relevant regions are binarized and a noise removal and thinning procedure is applied. This results in a skeletonized representation of the extracted regions. Ending points and bifurcation points of the skeletonized regions are represented by vertices. Additional vertices are inserted in regular intervals between ending points and bifurcation points. Finally, undirected edges are inserted to link vertices that are directly connected through a ridge in the skeleton. Each vertex is labeled with a two-dimensional attribute giving its position. Edges are attributed with an angle denoting the orientation of the edge with respect to the horizontal direction.

\subsection{Experimental Setup}

Setting of $L G Q$. We initialized the prototypes in a class-wise manner as follows: For every class, we applied the k-means algorithm for graphs proposed by [10]. To set the number $k$ and initialize the clusters, we partitioned the graphs according to their number of vertices. Each cell of the partition forms a cluster, if it contains at least $m$ graphs. The number $m$ was optimized with respect to the validation set. After applying k-means, we used the resulting cluster centers as initial prototypes of the LGQ algorithms.

We terminated both algorithms after $\max _{t}=100$ cycles through the training set. The learning rate was annealed according to $\eta_{t}=0.01 \cdot\left(1-t / \max _{t}\right)$, where 
$0 \leq t$ refers to the $t$-th cycle. For LGQ2.1 the window width was set to 0.1 for the letter and GREC data set and to 0.2 for the fingerprint data set.

Graph Distance Calculations and Optimal Alignment. For graph distance calculations and finding optimal alignments, we applied the graduated assignment algorithm [5]. This algorithm returns an approximate double-stochastic match matrix. We applied Munkres algorithm to convert the match matrix to a permutation sub-matrix. Using the permutation sub-matrix, we aligned the first graph towards the second.

Protocol. Both LGQ algorithms have been applied to the training set of each data set 10 times. To assess the generalization performance on the test sets, we have chosen the model that best predicts the class labels on the respective validation set. We compared the LGQ algorithms with the $\mathrm{kNN}$ method [15], the similarity kernel in conjunction with the SVM (SK+SVM) and the family of Lipschitz embeddings in conjunction with SVM (LE+SVM) proposed by [16] as well as the topological embedding approach based on the signature of a graph (TESG) proposed by [17.

\subsection{Results}

Table 2 summarizes the results. For TESG no results on letter (high) and fingerprint have been reported. Since LE+SVM refers to a family of related methods rather than a single method, Table 2 presents the best result over all methods of the LE+SVM family for each data set. In doing so, the comparison is biased towards LE+SVM.

The first observation to be made is that LGQ2.1 performs slightly superior than LGQ. Thus, as for feature vectors, pairwise adjustments of two prototypes belonging to different classes apparently better approximates the Bayes rule whereas LGQ tends to repel prototypes from Bayes decision surfaces in the graph space. The second observation to be made is that LGQ2.1 is comparable with the family of LE+SVM methods on GREC and fingerprint. Performance of LE+SVM family is, however, clearly superior on the letter data set.

As the results indicate, classifiers that directly operate in the domain of graphs can perform comparable to methods that embed graphs into vector spaces in order to apply state-of-the-art machine learning methods. An advantage of LVQ is its simplicity and efficiency. A simple initialization heuristic is sufficient to

Table 2. Classification accuracy (in \%) of LGQ and LGQ2.1

\begin{tabular}{lccc}
\hline \hline & Letter & GREC & Fingerprint \\
\hline kNN & 90.0 & 95.5 & 77.6 \\
SK+SVM & 79.1 & 94.9 & 41.0 \\
LE+SVM & 92.5 & 96.8 & 82.8 \\
TE & nil & 95.8 & nil \\
\hline LGQ & 80.9 & 94.7 & 79.2 \\
LGQ2.1 & 83.7 & 97.3 & 82.2 \\
\hline \hline
\end{tabular}


learn a relatively small number of prototypes, which, in addition, can be used to extract information about characteristic structural properties of a class.

\section{Conclusion}

Learning graph quantization generalizes LVQ by identifying attributed graphs with points in some Riemannian orbifold. The intrinsic metric of this orbifold turns out to be a generalized differentiable graph metric, which is widely used in a number of applications. The final step, to extend the update rule of LVQ for generalized differentiable distance function is straightforward and can be applied for distance spaces other than graphs. Despite its simplicity LGQ and LGQ2.1 performed comparable to state-of-the-art methods. The promising results suggest to extend generalized LVQ and soft LVQ to the domain of graphs.

\section{A Generalized Differentiable Functions}

Let $\mathcal{X}=\mathbb{R}^{n}$ be a finite-dimensional Euclidean space. A function $f: \mathcal{X} \rightarrow \mathbb{R}$ is generalized differentiable at $\boldsymbol{x} \in \mathcal{X}$ in the sense of Norkin [14] if there is a multi-valued map $\partial f: \mathcal{X} \rightarrow 2^{\mathcal{X}}$ in a neighborhood of $\boldsymbol{x}$ such that

1. $\partial f(\boldsymbol{x})$ is a convex and compact set;

2. $\partial f(\boldsymbol{x})$ is upper semicontinuous at $\boldsymbol{x}$, that is, if $\boldsymbol{y}_{i} \rightarrow \boldsymbol{x}$ and $\boldsymbol{g}_{i} \in \partial f\left(\boldsymbol{y}_{i}\right)$ for each $i \in \mathbb{N}$, then each accumulation point $\boldsymbol{g}$ of $\left(\boldsymbol{g}_{i}\right)$ is in $\partial f(\boldsymbol{x})$;

3. for each $\boldsymbol{y} \in \mathcal{X}$ and any $\boldsymbol{g} \in \partial f(\boldsymbol{y})$ holds $f(\boldsymbol{y})=f(\boldsymbol{x})+\langle\boldsymbol{g}, \boldsymbol{y}-\boldsymbol{x}\rangle+o(\boldsymbol{x}, \boldsymbol{y}, \boldsymbol{g})$, where the remainder $o(\boldsymbol{x}, \boldsymbol{y}, \boldsymbol{g})$ satisfies the condition

$$
\lim _{i \rightarrow \infty} \frac{\left|o\left(\boldsymbol{x}, \boldsymbol{y}_{i}, \boldsymbol{g}_{i}\right)\right|}{\left\|\boldsymbol{y}_{i}-\boldsymbol{x}\right\|}=0
$$

for all sequences $\boldsymbol{y}_{i} \rightarrow \boldsymbol{y}$ and $\boldsymbol{g}_{i} \in \partial f\left(\boldsymbol{y}_{i}\right)$.

We call $f$ generalized differentiable if it is generalized differentiable at each point $\boldsymbol{x} \in \mathcal{X}$. The set $\partial f(\boldsymbol{x})$ is the subdifferential of $f$ at $\boldsymbol{x}$ and its elements are called generalized gradients.

Generalized differentiable functions have the following properties [14]: 1. Generalized differentiable functions are locally Lipschitz and therefore continuous and differentiable almost everywhere. 2. Continuously differentiable, convex, and concave functions are generalized differentiable. 3. Suppose that $f_{1}, \ldots, f_{n}: \mathcal{X} \rightarrow$ $\mathbb{R}$ are generalized differentiable at $\boldsymbol{x} \in \mathcal{X}$. Then

$$
f_{*}(\boldsymbol{x})=\min \left(f_{1}(\boldsymbol{x}), \ldots, f_{m}(\boldsymbol{x})\right) \quad \text { and } \quad f^{*}(\boldsymbol{x})=\max \left(f_{1}(\boldsymbol{x}), \ldots, f_{m}(\boldsymbol{x})\right)
$$

are generalized differentiable at $\boldsymbol{x} \in \mathcal{X}$. 4. Suppose that $f_{1}, \ldots, f_{m}: \mathcal{X} \rightarrow \mathbb{R}$ are generalized differentiable at $\boldsymbol{x} \in \mathcal{X}$ and $f_{0}: \mathbb{R}^{m} \rightarrow \mathbb{R}$ is generalized differentiable at $\boldsymbol{y}=\left(f_{1}(\boldsymbol{x}), \ldots, f_{m}(\boldsymbol{x})\right) \in \mathbb{R}^{m}$. Then $f(\boldsymbol{x})=f_{0}\left(f_{1}(\boldsymbol{x}), \ldots, f_{m}(\boldsymbol{x})\right)$ is generalized differentiable at $\boldsymbol{x} \in \mathcal{X}$. The subdifferential of $f$ at $\boldsymbol{x}$ is of the form

$\partial f(\boldsymbol{x})=\operatorname{con}\left\{\boldsymbol{g} \in \mathcal{X}: \boldsymbol{g}=\left[\boldsymbol{g}_{1} \boldsymbol{g}_{2} \ldots \boldsymbol{g}_{m}\right] \boldsymbol{g}_{0}, \boldsymbol{g}_{0} \in \partial f_{0}(\boldsymbol{y}), \boldsymbol{g}_{i} \in \partial f_{i}(\boldsymbol{x}), 1 \leq i \leq m\right\}$. 
where $\left[\boldsymbol{g}_{1} \boldsymbol{g}_{2} \ldots \boldsymbol{g}_{m}\right]$ is a $(N \times m)$-matrix. 5. Suppose that $F(\boldsymbol{x})=\mathbb{E}_{\boldsymbol{z}}[f(\boldsymbol{x}, \boldsymbol{z})]$, where $f(\cdot, \boldsymbol{z})$ is generalized differentiable. Then $F$ is generalized differentiable and its subdifferential at $\boldsymbol{x} \in \mathcal{X}$ is of the form $\partial F(\boldsymbol{x})=\mathbb{E}_{\boldsymbol{z}}[\partial f(\boldsymbol{x}, \boldsymbol{z})]$.

\section{References}

1. Almohamad, H., Duffuaa, S.: A linear programming approach for the weighted graph matching problem. IEEE Transactions on PAMI 15(5), 522-525 (1993)

2. Caetano, T.S., Cheng, L., Le, Q.V., Smola, A.J.: Learning graph matching. In: ICCV (2007)

3. Cour, T., Srinivasan, P., Shi, J.: Balanced graph matching. In: NIPS (2006)

4. Dosch, P., Valveny, E.: Report on the second symbol recognition contest. In: Liu, W., Lladós, J. (eds.) GREC2005.LNCS, vol. 3926,pp.381-397. Springer,Heidelberg (2006)

5. Gold, S., Rangarajan, A.: Graduated Assignment Algorithm for Graph Matching. IEEE Transactions on PAMI 18, 377-388 (1996)

6. Gold, S., Rangarajan, A., Mjolsness, E.: Learning with preknowledge: clustering with point and graph matching distance measures. Neural Computation 8(4), 787-804 (1996)

7. Günter, S., Bunke, H.: Self-organizing map for clustering in the graph domain. Pattern Recognition Letters 23(4), 405-417 (2002)

8. Hammer, B., Strickert, M., Villmann, T.: Supervised neural gas with general similarity measure. Neural Processing Letters 21(1), 21-44 (2005)

9. Jain, B., Wysotzki, F.: Central Clustering of Attributed Graphs. Machine Learning 56, 169-207 (2004)

10. Jain, B., Obermayer, K.: Structure Spaces. Journal of Machine Learning Research 10, 2667-2714 (2009)

11. Kohonen, T.: Self-organizing maps. Springer, Heidelberg (1997)

12. Kohonen, T., Somervuo, P.: Self-organizing maps of symbol strings. Neurocomputing 21(1-3), 19-30 (1998)

13. Neuhaus, M., Bunke, H.: A graph matching based approach to fingerprint classification using directional variance. In: Kanade, T., Jain, A., Ratha, N.K. (eds.) AVBPA 2005. LNCS, vol. 3546, pp. 191-200. Springer, Heidelberg (2005)

14. Norkin, V.I.: Stochastic generalized-differentiable functions in the problem of nonconvex nonsmooth stochastic optimization. Cybernetics 22(6), 804-809 (1986)

15. Riesen, K., Bunke, H.: IAM Graph Database Repository for Graph Based Pattern Recognition and Machine Learning. In: da Vitoria Lobo, N., Kasparis, T., Roli, F., Kwok, J.T., Georgiopoulos, M., Anagnostopoulos, G.C., Loog, M. (eds.) S+SSPR 2008. LNCS, vol. 5342, pp. 287-297. Springer, Heidelberg (2008)

16. Riesen, K., Bunke, H.: Graph Classification by Means of Lipschitz Embedding. IEEE Transactions on Systems, Man, and Cybernetics 39(6), 1472-1483 (2009)

17. Sidere, N., Heroux, P., Ramel, J.-Y.: Vector Representation of Graphs: Application to the Classification of Symbols and Letters. In: Conference Proceedings on Document Analysis and Recognition, pp. 681-685 (2009)

18. Sumervuo, P., Kohonen, T.: Self-organizing maps and learning vector quantization for feature sequences. Neural Processing Letters 10(2), 151-159 (1999)

19. Umeyama, S.: An eigendecomposition approach to weighted graph matching problems. IEEE Transactions on PAMI 10(5), 695-703 (1988)

20. Watson, C., Wilson, C.: NIST Special Database 4, Fingerprint Database. National Institute of Standards and Technology (1992)

21. Van Wyk, M., Durrani, M., Van Wyk, B.: A RKHS interpolator-based graph matching algorithm. IEEE Transactions on PAMI 24(7), 988-995 (2002) 